\title{
Commentary
}

\section{Eradication of foot-and-mouth disease: a foot in mouth proposition}

Foot-and-mouth disease (FMD) is the most contagious disease of cloven-hoofed animals. The disease is highly infectious and is characterized by rapid transmission, a sequel to the rather explosive growth cycle of the causative virus, FMDV (an aphthovirus; Picornaviridae). As few as 10-25 virus particles can establish air-borne infection in domestic ruminants and pigs. The latter emit the greatest amounts of virus as aerosols. In unvaccinated herds mortality can be high, particularly in young cattle and pigs. Milk production ceases and animals used for traction can become useless. Internationally agreed policy dictates that once a country has adopted an eradication strategy, all animals on affected farms must be destroyed. In areas where the disease occurs, trade of livestock and animal products must be blocked, and a wide range of agricultural products may be banned from export to other countries.

FMDV is a positive strand RNA virus whose genomic RNA is embedded in 60 copies of each of the four viral structural proteins, VP1-4. The surface of the virus has several parts of VP1, VP2 and VP3 as revealed by monoclonal antibody binding studies and X-ray crystallography. The three capsid proteins have similar tertiary structures and form an eight-stranded $\beta$-barrel. Parts of the loops between the $\beta$-strands are exposed at the surface of the virus particle and contribute to the antigenic site. One of the loops in VP1, the so-called FMDV loop (residues 140-160), forms a major antigenic site. There are seven serotypes of FMDV: A, O, C, Asia 1 and the South African types SAT 1, 2 and 3. The classical European serotypes are types $\mathrm{A}, \mathrm{O}$ and $\mathrm{C}$ which have spread to other continents, probably due to importation of European cattle. Serotype A viruses are the most variable viruses, having more than 30 subtypes. Some of them are strongly divergent and vaccines made against one subtype may only protect against the others after repeated vaccinations (Barteling and Vreeswijk 1991). Chemically inactivated and adjuvanated virus grown in cell culture (viral antigens) can be used to generate vaccines which induce sufficient levels of virus-neutralizing antibodies. The immunizing moiety in FMD vaccine is the intact 140 S particle. Immunological protection against FMD correlates with the presence of antibodies in the serum which neutralize virus infectivity.

Since the end of 1960s, Western Europe has been virtually free of FMD. Occasional outbreaks have occurred locally. North and Central America, Australia, New Zealand, Japan, Indonesia and some Asian countries are free of the disease. FMD is still a major problem in West Asia, in Southeast Asia, in Africa and on the South American continent with the exception of Chile. In recent months, FMD has created havoc in Great Britain. According to information released by the UK Government in April 2001, the total number of animals slaughtered or identified as being for slaughter is around 1.2 million and more than 1000 animals have been confirmed to have the disease (MAFF; http://www.maff.gov.uk).

The magnitude of this mass slaughter of animals in the wake of an outbreak raises several issues. Firstly, there is nothing like 'total eradication' of any disease from a given country or region, if the virus is lurking in any form at any remote corner of the world. The virus movement could gain greater momentum with increased mobilities of people and speed of transportation of animals from endemic areas. Given the complexities of the evolution of RNA viruses and their survival strategies, the time has come now to question the policy of banning vaccination. The country which is opting to slaughter all suspected animals, a policy which could be termed 'inhuman', could have made a national policy of vaccination as the protection mechanism against FMD. However, vaccination is not a policy of any EU member state or the European Commission; the UK government too is not prepared to be removed from its so-called "disease-free status", which is just what vaccination would be taken to imply. The loss of disease-free status is expected to cripple exports currently worth more than 1.3 billion pounds per year. 
This brings to focus the second influence on human logic - greed. Each developed nation competes with another for having an upper hand on the global market. The economics of world trade seems to be driving governments and policy makers to stop vaccination and declare their country free of a particular animal disease. Under these circumstances, it appears that new generation vaccines (without any virus particles) have to be developed and these have to totally protect vaccinated animals. This could eventually lead to the acceptance of emergency vaccination of susceptible animals instead of mass killing.

\section{References}

Barteling S J and Vreeswijk J 1991 Developments in foot-and-mouth disease vaccines; Vaccine 9 75-88

M S SHAILA

Department of Microbiology and Cell Biology, Indian Institute of Science,

Bangalore 560 012, India

(Email,shaila@mcbl.iisc.ernet.in) 


\section{Understanding intercellular communication in the brain: Identified neuromuscular synapses of the fruitfly Drosophila serve as a model}

The transmission of information between nerve cells in the brain takes place at specialized sites of contact, the synapses. Spatial interactions between synapses and temporal modulation of synaptic efficacy represent basic functional elements required for all higher brain functions including feature abstraction, learning and memory, and cognition. The detailed analysis of the molecular mechanisms which control and modify synaptic transmission is thus of fundamental significance for understanding brain function in health and disease and has therefore become a focal research area in the neurosciences. At chemical synapses, which constitute the predominant type of contacts, electrical signals of the presynaptic neuron cause the release of neurotransmitters and neuromodulators which diffuse to the postsynaptic cell and modify either its membrane potential or intracellular chemical reactions, or both. Structurally, synaptic contact sites are characterized on the presynaptic side by an accumulation of synaptic vesicles and a membrane thickening, often associated with ribbon-shaped specializations, a synaptic cleft that separates pre- and postsynaptic cell, and again a membrane thickening on the postsynaptic side. Depolarization of the presynaptic membrane causes the opening of voltage-sensitive calcium channels which allow calcium to enter the presynaptic terminal. This calcium signal induces the transmitter-loaded synaptic vesicles to fuse with the presynaptic membrane (exocytosis) and to shed their content into the synaptic cleft. Binding of the transmitter molecules to receptor proteins embedded in the postsynaptic membrane modulates ionic currents across the postsynaptic membrane and/or triggers intracellular signalling cascades with a wide range of possible effects on metabolic reactions modifying proteins and altering gene expression. The synaptic vesicle membrane, which by exocytosis had become integrated into the presynaptic membrane, is taken up back into the presynaptic terminal to form new synaptic vesicles by the process of endocytosis (synaptic vesicle recycling). The basic principles involved in synaptic transmission have been known for almost half a century, and a few dozen proteins specifically expressed at the synapse have been identified in recent years. However, the detailed molecular events leading to calcium-triggered exocytosis and endocytotic recycling of synaptic vesicles are still largely unknown.

It is clear that the study of the concerted interactions of protein complexes involved in exo-endocytotic synaptic vesicle cycling greatly profits from the availability of genetic tools. The fruitfly Drosophila has been a favourite object of genetic studies for almost a century, main reasons being easy culturing which allow the maintenance of thousands of mutant strains in a small space, short generation period of 10 days, and the giant chromosomes of larval salivary glands. Modern gene technology now permits single proteins to be eliminated by genetic knock-out and to be re-introduced by transgenic techniques after specific modifications by site-directed in vitro mutagenesis of the encoding genes. Genetic dissection of the molecular mechanisms involved in synaptic vesicle cycling in fact has made extensive use of Drosophila in order to assay the effects of the genetic changes in an otherwise intact organism. In recent years a large collection of mutants has been assembled which affect a wide variety of synaptic proteins and help to shape the evolving picture of the molecular basis of neurotransmitter release and vesicle recycling.

Since a single neuron in the brain often receives synaptic input from hundreds of presynaptic cells and in turn transmits its signals to similar numbers of postsynaptic neurons, vesicle trafficking in these synapses is exceedingly difficult to study. Rather, most information on synaptic function has been obtained by the study of certain identified synapses in model systems such as the frog neuromuscular junction, the mammalian cervical ganglion, the squid giant synapse, and, more recently the larval neuromuscular junction of Drosophila. This latter system had been established 25 years ago (Jan and Jan 1976) but has recently become highly attractive by the application of fluorescent dyes and the use of conditional mutations which block synaptic transmission at high but not at low temperature.

When supplied in the extracellular fluid, the fluorescent dye FM1-43 is taken up into synaptic vesicles during endocytosis and thus selectively stains vesicles that have been recycled. These vesicles 
destain only after renewed exocytotic fusion (transmitter release). Thus it is possible to image the entire exo-endocytotic synaptic vesicle cycle in vivo at high spatial and temporal resolution.

In a Drosophila mutant (shibire-ts) that had been isolated due to its reversible paralysis at high temperatures the GTPase enzyme dynamin is modified. Dynamin is involved in endocytotic vesicle recycling. In the mutant endocytosis is blocked at non-permissive temperatures. Using this mutant the dynamics of vesicle depletion and replenishment in the synapses of the larval neuromuscular junction has now been studied quantitatively in a series of elegant experiments (Estes et al 1996; Koenig and Ikeda 1996; Kuromi and Kidokoro 1998, 1999, 2000; Delgado et al 2000).

The larval body wall of Drosophila contains sets of 30 muscles stereotypically repeated from body segment to body segment. In a larva cut open along the dorsal midline, the muscle pairs 6/7 and their innervating motorneurons are easily recognized and accessible to optical and electrophysiological analysis. Muscles 6/7 are innervated by 2-4 motorneurons whose terminal branches invade the muscle and form characteristic varicose thickenings along their length, termed synaptic boutons. These boutons contain several presynaptic specializations (membrane thickening with T-shaped ribbon surrounded by a cluster of synaptic vesicles) adding up to a total of about 550 transmitter release sites on each muscle (Atwood et al 1993). By two-electrode voltage clamp recordings from the muscle and stimulation of the motor neuron it has recently been possible to follow the time course of vesicle fusion during prolonged stimulation (Delgado et al 2000).

In a first experiment the authors decreased external calcium concentrations to such low values that the muscle responds only to a small percentage of the stimuli. Under these conditions most responses that are obtained will represent the effect of the fusion of a single vesicle. In this way the electric charge entering the muscle in response to the transmitter content of a single vesicle (unitary stimulus) can be determined. By dividing the total charge transfer across the muscle membrane due to a stimulus under normal calcium conditions by this unitary charge, the number of vesicles released by such a normal stimulus can be calculated.

When a long train of stimulus pulses is applied, the number of vesicles released per pulse at first decreases sharply, then more slowly, and finally a steady state is reached where the number of released vesicles equals the number of recycled vesicles. This number depends on the stimulus frequency for low stimulus rates but saturates at about 1000 vesicles per $\mathrm{s}$ at $10 \mathrm{~Hz}$ indicating that the maximum recycling rate at each release site is about 1.8 vesicles per $\mathrm{s}$. The data demonstrate the existence of three distinct pools of synaptic vesicles, a small pool that is immediately releasable (IRP), an intermediate readily releasable pool (RRP), and a reserve pool (RP) that is activated only during highfrequency stimulation. Using the shi-ts mutant at non-permissive temperature (no recycling) the capacities of these pools has been determined. The IRP is depleted to 1/e (37\%) after 2-4 stimuli, the RRP after 100 to 200 stimuli, while the RP reaches this level after 1500-2500 stimuli. At high stimulus frequencies the RRP and RP are depleted by fewer stimuli possibly due to some process of facilitation. From these experiments the total number of synaptic vesicles within the boutons on muscle 6 or 7 has been calculated to be about 90,000 .

Using the fluorescent dye FM1-43 the position of the vesicle pools within each synaptic bouton could be identified. The vesicles of the RRP locate to the periphery of the boutons while the RP is concentrated towards the centre of the bouton. The rates of destaining of the bouton periphery corresponded well to the electrophysiologically determined depletion of the RRP while the destaining of the centre of the bouton reflected the depletion of the reserve pool. By these experiments it was verified that endocytosis is blocked in shi-ts mutants at non-permissive temperatures. In addition it was demonstrated, however, that translocation of vesicles from the releasable pools to the reserve pool becomes negligible under these conditions. The reverse, recruitment of reserve pool vesicles to the RRP, can be largely inhibited by treating the preparation with cytochalasin-D, a drug that blocks actin polymerization. Using this drug the authors could estimate that the RRP consists of about $14 \%$ to $20 \%$ of the total number of vesicles.

From these experiments the observed quantal content of each of a train of repetitive stimuli could be calculated by a simple sum of three exponential functions. It will be interesting to see if the predictive power of the equations also hold true for less regular (more natural) stimulus volleys. As is generally the case in biology, one also has to expect further complications due to the actual complexity of the system. There are at least three types of boutons on muscles 6 or 7 , with differences in structure, 
vesicle content, and protein composition. It is unlikely that these differences should play no role in over-all synaptic function. Nontheless the experiments described represent an important step towards quantitative modelling of synaptic transmission which eventually, when similar data become available for the considerably more heterogeneous interneuronal synapses (Stevens and Wesseling 1998), may lead to a better understanding of the behaviour of networks of neurons in the brain.

\section{References}

Atwood H L, Govind C K and Wu C-F 1993 Differential ultrastructure of synaptic terminals of ventral longitudinal abdominal muscles in Drosophila larvae; J. Neurobiol. 24 1008-1024

Delgado R, Maureira C, Oliva C, Kidokoro Y and Labarca P 2000 Size of vesicle pools, rates of mobilization, and recycling at neuromuscular synapses of a Drosophila mutant, shibire; Neuron 28 941-953

Estes P S, Roos J, van der Bliek A, Kelly R B, Krishnan K S and Ramaswami M 1996 Traffic of dynamin within individual Drosophila synaptic boutons relative to compartment-specific markers; J. Neurosci. 16 5443-5456

Jan L Y and Jan Y N 1976 Properties of the larval neuromuscular junction in Drosophila melanogaster; J. Physiol. (London) 262 189-214

Koenig J H and Ikeda K 1996 Synaptic vesicles have two distinct recycling pathways; J. Cell Biol. 135 797-808

Kuromi H and Kidokoro Y 1998 Two distinct pools of synaptic vesicles in single presynaptic boutons in a temperature-sensitive Drosophila mutant, shibire; Neuron 20 917-925

Kuromi H and Kidokoro Y 1999 The optically determined size of exo/endo cycling vesicle pool correlates with the quantal content at the neuromuscular junction of Drosophila larvae; J. Neurosci. 19 1557-1565

Kuromi H and Kidokoro Y 2000 Tetanic stimulation recruits vesicles from reserve pool via a cAMP-mediated process in Drosophila synapses; Neuron 27 133-143

Stevens C F and Wesseling J F 1998 Activity-dependent modulation of the rate at which synaptic vesicles become available to undergo exocytosis; Neuron 21 415-424

ERICH BUCHNER

Department of Biological Sciences, Tata Instiute of Fundamental Research, Mumbai 400 005, India

(Email: buchner@biozentrum.uni-wuerzburg.de) 


\section{Cell fate choice and social evolution in Dictyostelium discoideum: Interplay of morphogens and heterogeneities}

Attempts to understand the development of the social amoeba Dictyostelium discoideum keep throwing up surprises and drive home the point that here too, as in any biological situation, no explanation can make sense except in the light of evolution.

The life cycle of all cellular slime moulds (Dictyostelids) consists of two distinct phases, growth and development. During the period of growth, single amoebae that have germinated from different spores feed on bacteria and go through mitotic divisions until the food supply is exhausted. The developmental phase is triggered and maintained by starvation. Starved cells stop dividing, aggregate via chemotaxis and form a multicellular, motile, 'embryonic' mass, the slug (typically a tapered cylinder of about $1 \mathrm{~mm} \times 50 \mu \mathrm{m})$. Within the slug of $D$. discoideum a clear cellular fate map can be discerned (Bonner 1952). Presumptive stalk (pst) cells occupy about the anterior $20 \%$ of the slug and presumptive spore (psp) cells the remaining 80\% (MacWilliams and Bonner 1979); the long-held belief that the relative proportions of pst and psp cells are tightly regulated has been questioned very recently (Rafols et al 2001). Gene expression patterns enable a further division of the pst zone into an anterior pstA region and a posterior pstO region (Williams et al 1989), with the cells in the two regions corresponding to different functional classes. Thus, there is a division of labour within the slug - as it happens, in a literal sense too, because the motive force for slug movement is provided by pst cells (Inouye and Takeuchi 1980). After a facultative migratory phase, the slug transforms into a fruiting body comprising a ball of spores atop a dead cellular stalk (Bonner 1967).

How do the members of a seemingly homogenous cell population come to adopt diverse fates? An attractive hypothesis suggested itself early. It was fuelled by three considerations. Firstly, Raper (1940) showed in an elegant experiment that isolated pst or psp fragments can regulate. That is, they restore the missing cell type and go on to form normally proportioned fruiting bodies (albeit only after a period of migration in the case of pst isolates). Therefore, at least under some conditions, intercellular interactions within the slug are necessary for generating both cell types in the correct ratio. Secondly, the fate map in the slug seemed to exhibit an unambiguous anterior-posterior spatial pattern. And thirdly, on theoretical grounds, such a pattern made it attractive to think of a spontaneously arising linear gradient of morphogen concentration as being responsible for the fate map. It appeared natural to assume that an amoeba would eventually become a stalk cell or a spore depending on where it found itself within the gradient. The hope even arose that this might be the long-sought example of a Turing system in biology.

Studies that began over two decades ago made it seem plausible that one or the other member of a family of chlorinated hexaphenones made by developing amoebae could be the morphogen (Town et al 1976; Morris et al 1987). These were collectively termed DIF for Differentiation-Inducing Factor. DIF could induce stalk differentiation and inhibit spore differentiation. DIF-1, the most potent isoform of DIF, was chemically synthesized and shown to stimulate the expression of pst genes and inhibit the expression of psp genes (reviewed by Kay et al 1999). Based on the observation that pstA cells required a 10-fold higher concentration of DIF-1 than pstO cells (Early et al 1995), it was thought that varying levels of DIF-1 also induced different pst cell types. Strikingly, and in retrospect misleadingly, a mutant (HM44) that failed to make DIF did not develop beyond the tipped mound stage and formed no stalk cells, even under in vitro stalk cell induction conditions (Kopachik et al 1983).

But soon the picture started looking murky. For one thing, while there was indeed a spatial gradient of DIF-1 in the slug, it was the wrong way round. Psp cells turned out to contain more DIF-1 activity than pst cells, in fact about twice as much (Brookman et al 1987); unpublished results cited in Thompson and Kay (2000b) indicate that DIF-1 may be produced largely by psp cells. The shape of the gradient was later accounted for by the finding that the activity of an enzyme that degraded DIF-1, DIF-1 dechlorinase, was concentrated in the anterior of the slug (Kay et al 1993). (The possibility remains open that what is meaningful is the amount of DIF-1 tightly bound to an intermediate in the signal transduction pathway - as opposed to the amount of free DIF-1 - and that its level might indeed 
be higher in the pre-stalk region). Another problem was that not all pst genes tested were capable of being induced by DIF-1 (Shaulsky and Loomis 1996). Recent work has gone further: it appears that the primary signal for cell type choice cannot be based on spatial gradients of DIF at all (Thompson and Kay 2000a, b). Rather, the choice of cell type appears to rest on a basis that is quite the opposite of morphogen-dependent spontaneous patterning, namely the existence of functional differences, in the form of pre-existing heterogeneities, between the members of an apparently homogeneous cell mass (Bonner 1952; Takeuchi 1969).

The evidence is growing that DIF- (or DIF-1)-related properties are one among the many indicators of early heterogeneity in D. discoideum. An aspect of DIF-1 related heterogeneity was demonstrated by Azhar et al (1997) using fluorescence-activated cell sorting (FACS). They monitored the relative distribution of "low calcium" (psp) and "high calcium" (pst) cells in pre- or post-aggregation amoebae and found that DIF-1 stimulation caused an increase in intracellular calcium only in a subset of amoebae. All of these fell in the low calcium or psp class; and, as a result of the calcium increase, entered the putative pst class (the stimulus may have also reinforced the status of pst cells but the experiment was incapable of demonstrating this). This observation raised the possibility that DIF-1 influenced cell differentiation by acting on amoebae that were already different in terms of how sensitive they would be to it. That possibility has now been verified by Thompson and Kay (2000a). They show that the manner in which amoebae respond to DIF-1 depends on their prior nutritional status, thereby supporting a conjecture based on an evolutionary argument (Atzmony et al 1997). In terms of their tendency to adopt a pst fate, cells that are raised in the absence of added glucose are more sensitive to DIF-1 than cells that are raised in a glucose-rich environment. A parallel pattern of responsiveness to DIF-1 is seen when cells that are starved early in the cell cycle are compared to those starved when late in the cell cycle (Thompson and Kay 2000a).

Astonishingly, it turns out that $D$. discoideum can develop normally - to a degree - in the absence of any detectable DIF-1. Thompson and Kay (2000b) have generated a strain (dmtA) lacking the des-methyl-DIF-1methyl transferase gene, the gene that encodes the enzyme which catalyses a key step in the DIF-1 biosynthetic pathway. The resulting DIF-1 ${ }^{-}$amoebae aggregated with a slight delay in comparison to wild type and the fruiting bodies produced few spores. Though the slugs often broke up or laid down a stalk while migrating, the spatial and temporal expression profile of several cell typespecific genes was similar to that of the wild type. In particular, DIF-1 was sufficient but not necessary for the induction of ecmA (pstA cell-specific) gene expression: ecmA transcripts appeared not only on schedule but also in the correct location in the DIF- $1^{-}$slugs. The absence of DIF-1 had an additional consequence. $d m t A$ slugs lacked pstO cells, implying that DIF-1 might have been needed only for the differentiation of this particular pst sub-type. The pstO defect could be restored by supplying exogenous DIF-1 via the agar substratum, suggesting that a spatial gradient of DIF-1 is not essential for normal patterning (explicit measurements will be needed before it can be ruled out that the net intake of DIF-1 varies along the length of the slug).

These results once again raise the question of how cell fate is decided in D. discoideum. Inevitably, they re-direct attention towards intercellular heterogeneity as the primary factor behind cell fate choice. Pre-aggregation amoebae can differ in many ways. These include nutritional status, cell size, cell cycle phase at starvation, cellular calcium content (reviewed in Nanjundiah 1997) and, now, sensitivity to DIF-1. All act as inputs that influence the tendency of a cell to become pst or psp, but that tendency or bias can be translated into a decision only in the context of the inputs operating on other cells. Obviously, this means that a cell must be able to communicate with others and be capable of assessing another's bias relative to its own. The point is that intercellular communication-based differentiation and cell-autonomous cell type choice should not be thought of in either/or terms. At times the issue has been addressed in terms of position-dependent fate determination versus fate-dependent position determination. But the dichotomy is a false one (Bonner 1992).

In other words, the determination of cell fate involves a subtle and non-exclusive interplay between cell-autonomous factors and intercellular signalling. Nevertheless, why do most experiments on D. discoideum yield results indicating one or the other mechanism? The answer must be sought in the evolutionary history and ecology of Dictyostelid amoebae in general and D. discoideum in particular. Let us suppose that the ancestors of modern Dictyostelids evolved as clonally propagating populations - meaning that aggregates were mostly clonal. In that case, one can see how decisions 
pertaining to division of labour that were advantageous to the (clonal) group as a whole would be favoured. Such decisions would have to be taken communally and would of necessity involve cell-tocell signalling. The nature of the signals could be wholly arbitrary, dictated solely by convention. At the other extreme, suppose genetically heterogeneous aggregates were, and are, common in the wild. Then individual-level selection, rather than group selection (at the level of the clonal or kin group), would be expected to operate. The propensity on the part of a cell to adopt one or the other fate would hinge on factors intrinsic to the cell. These factors can be thought of as fitness-related qualities that depended on the cell's genotype as well as phenotype. Of course the factors would come into play only in relation to similar factors in the other cells in an aggregate. The means used by cells, both for communicating their own qualities and assessing the qualities of others, could no longer involve arbitrary signals. Rather, the signals would have to be reliable indicators of quality (Atzmony et al 1997). This analysis overlaps with the between-cell versus within-cell distinction drawn by Bonner (1992).

A history of clonal propagation and conventional intercellular signals, or a history of mixed aggregation and intrinsic factors, represent extreme alternatives. A less restrictive hypothesis would be to posit an evolutionary history in which Dictyostelium amoebae were sometimes members of clonal aggregates and at other times members of genetically heterogeneous aggregates. In that case one might think that natural selection would have favoured a mixed strategy wherein an amoeba could, depending on the circumstances, base its behaviour either on arbitrary signals or on signals that conveyed cellular quality. But even if Dictyostelid ancestors had always participated in clonal aggregates, phenotypic heterogeneity would be unavoidable. Individual-level selection would make it more likely that (relatively) high quality amoebae would form spores and (relatively) low quality amoebae would contribute to the stalk.

In the light of these considerations, how might one assess the developmental role of DIF-1? The production of, and sensitivity to, DIF-1 could be just one of the many measures of cellular quality (Atzmony et al 1997). In its absence, cells would still be capable of assessing each others' qualities. It is important to stress that what we have discussed pertains to a restricted set of facts. Integrating everything that is known regarding cellular behaviour in $D$. discoideum into a coherent evolutionary picture will be an enormously complex undertaking. Experiments directed towards understanding the natural history of cellular slime moulds should provide us with important clues behind the logic underlying cell fate choice.

\section{References}

Atzmony D, Zahavi A and Nanjundiah V 1997 Altruistic behaviour in Dictyostelium discoideum explained on the basis of individual selection; Curr. Sci. 72 142-145

Azhar M, Kennady P K, Pande G and Nanjundiah V 1997 Stimulation by DIF causes an increase of intracellular $\mathrm{Ca}^{2+}$ in Dictyostelium discoideum; Exp. Cell Res. $230403-406$

Bonner J T 1952 The pattern of differentiation in amoeboid slime molds; Am. Nat. 86 79-89

Bonner J T 1967 The cellular slime molds Second edition (Princeton: Princeton University Press)

Bonner J T 1992 The fate of a cell is the function of its position and vice-versa; J. Biosci. 17 95-114

Brookman J J, Jermyn K A and Kay R R 1987 Nature and distribution of the morphogen DIF in the Dictyostelium slug; Development 100 119-124

Early A E, Abe T and Williams J G 1995 Evidence for positional differentiation of prestalk cells and for a morphogenetic gradient in Dictyostelium; Cell 83 91-99

Inouye K and Takeuchi I 1980 Motive force of the migrating pseudoplasmodium of the cellular slime mould Dictyostelium discoideum; J. Cell Sci. 41 53-64

Kay R R, Large S, Traynor D and Nayler O 1993 A localized differentiation-inducing-factor sink in the front of the Dictyostelium slug; Proc. Natl. Acad. Sci. USA 90 487-491

Kay R R, Flatman, P and Thompson, C R L 1999 DIF signaling and cell fate; Sem. Cell Dev. Biol. 10 577-585

Kopachik W, Oohata B, Dhokia B, Brookman J J and Kay R R 1983 Dictyostelium mutants lacking DIF, a putative morphogen; Cell 33 397-403

MacWilliams H K and Bonner J T 1979 The prestalk-prespore pattern in cellular slime molds; Differentiation 14 $1-22$

Morris H R, Taylor G W, Masento M S, Jermyn K A and Kay R R 1987 Chemical structure of the morphogen differentiation-inducing factor from Dictyostelium discoideum; Nature (London) 328 811-814

Nanjundiah V 1997 Models for pattern formation in the Dictyostelid slime molds; in Dictyostelium: A model system for cell and developmental biology (eds) Y Maeda, K Inouye and I Takeuchi (Tokyo: Universal Academy Press) pp 305-316 
Rafols I, Amagai A, Maeda Y, MacWilliams H K, Sawada Y 2001 Cell type proportioning in Dictyostelium slugs: lack of regulation within a $2 \cdot 5$-fold tolerance range; Differentiation (in press)

Raper K B 1940 Psuedoplasmodium formation and organisation in Dictyostelium discoideum; J. Elisha Mitchell Sci. Soc. 56 241-282

Shaulsky G and Loomis W F 1996 Initial cell type divergence in Dictyostelium is independent of DIF-1; Dev. Biol. 174 214-220

Takeuchi I 1969 in Nucleic acid metabolism: cells differentiation and cancer growth (eds) E F Cowdry and S Seno (Oxford: Pergamon Press) pp 297-304

Thompson C R L and Kay R R 2000a Cell fate choice in Dictyostelium: intrinsic biases modulate sensitivity to DIF signaling; Dev. Biol. 227 56-64

Thompson C R L and Kay R R 2000b The role of DIF-1 signaling in Dictyostelium development; Mol. Cell 6 $1509-1514$

Town C D, Gross, J D and Kay R R 1976 Cell differentiation without morphogenesis in Dictyostelium discoideum; Nature (London) 262 717-719

Williams J G, Jermyn K A and Duffy K T 1989 Formation and anatomy of the prestalk zone of Dictyostelium; Development (Suppl.) 107 91-97

TRUPTI S KAWLI

SONIA KAUSHIK*

Department of Molecular Reproduction, Development and Genetics and

*Center for Ecological Sciences,

Indian Institute of Science,

Bangalore 560 012, India

(Email,trupti@mrdg.iisc.ernet.in) 